\title{
Top quark physics with the ATLAS detector: recent highlights
}

\author{
N Bruscino ${ }^{1}$, on behalf of the ATLAS Collaboration \\ ${ }^{1}$ INFN Sezione di Roma and Dipartimento di Fisica, Sapienza Università di Roma, \\ Roma; Italy. \\ E-mail: nello.bruscino@cern.ch, nello.bruscino@roma1.infn.it
}

\begin{abstract}
The top quark is the heaviest known fundamental particle. As it is the only quark that decays without hadronisation, it provides the unique opportunity to probe the properties of bare quarks at the Large Hadron Collider (LHC). This article will present highlights of a few recent precision measurements of the top quark using $13 \mathrm{TeV}$ collision data with the ATLAS experiment. Top-quark pair and single top production cross sections, including differential distributions and production in association with bosons, will be presented alongside top quark properties measurements. Measurements of the top-quark mass and searches for rare top decays are also presented.
\end{abstract}

Keywords: top quark, ATLAS, high energy physics

Submitted to: Phys. Scr. 


\section{Introduction}

The large mass of the top quark, which is close to the electroweak symmetry breaking scale, indicates that this particle could play a special role in the Standard Model (SM) as well as in beyond the Standard Model (BSM) theories. Moreover, the top quark has a very short lifetime $\left(\tau=0.5 \times 10^{-25} \mathrm{~s}\right)$ and decays before hadronisation $\left(\tau_{\text {had }} \sim 10^{-23} \mathrm{~s}\right)$ or spin de-correlation take place $\left(\tau_{\text {spin dec. }} \sim 10^{-22} \mathrm{~s}\right)$. Therefore several properties of the top quark may be measured precisely from its decay products.

Due to the high top-pair production $(t \bar{t})$ cross section for $13 \mathrm{TeV}$ proton-proton $(p p)$ collisions, the Large Hadron Collider (LHC) experiments collect an unprecedented number of top-quark events. The copious amount of detected events allows for high precision measurements in order to probe predictions of quantum chromodynamics (QCD), which provides the largest contribution to $t \bar{t}$ production. This process may also reveal BSM particles with a large coupling to the top quark.

This note describes the most recent results in the top-quark sector by the ATLAS [1] Collaboration. It consists of four sections:

- latest cross-section measurements;

- state-of-the-art measurement of the top mass;

- measurements of the properties of the top quark (spin correlation, charge asymmetry, $\left.\left|V_{t b}\right|, \ldots\right)$;

- latest results on the associated production of top-quark pairs with additional heavyflavour jets;

- search for new physics produced in association with top quarks;

- measurements of strange hadron production from $t \bar{t}$ pair decays.

\section{Cross Section measurements at $\sqrt{s}=13 \mathrm{TeV}$}

\subsection{Measurements of top-quark pair differential and double-differential cross-sections} in the $\ell+j e t s$ channel

The measurement of single- and double-differential cross-sections at $\sqrt{s}=13 \mathrm{TeV}[2]$, performed by the ATLAS Collaboration, probe the top-quark kinematic properties at this centre-of-mass energy and complement recent measurements involving leptonic final states by ATLAS and CMS. All the measurements are performed in the selected fiducial phase-spaces and the results extrapolated to the full phase-space at the parton level.

In the SM, the top quark decays almost exclusively into a $W$ boson and a $b$ quark. The signature of a $t \bar{t}$ decay is therefore determined by the $W$ boson decay modes. This analysis makes use of the semileptonic decay $\ell+$ jets of the $t \bar{t}$ pair. In this case one $W$ boson decays into an electron or a muon and a neutrino, and the other $W$ boson decays into a quark-antiquark pair, with the two decay modes referred to as the $e+$ jets and $\mu+$ jets channels, respectively. Events in which the $W$ boson decays into an electron or muon through a $\tau$-lepton decay may also meet the selection criteria. Since 
the reconstruction of the top quark depends on its decay products, two top quarks are referred to as 'hadronically (or leptonically) decaying top quarks' (or alternatively 'hadronic/leptonic top'), depending on the $W$ boson decay mode.

Two complementary topologies of the $t \bar{t}$ final state in the $\ell+$ jets channel are exploited, referred to as 'resolved' and 'boosted', where the decay products of the hadronically decaying top quark are either angularly well separated or collimated into a single large-radius jet reconstructed in the calorimeter, respectively. As the jet selection efficiency of the resolved analysis decreases with increasing top-quark transverse momentum, the boosted selection allows events with higher-momentum hadronically decaying top quarks to be efficiently selected.

Since the $t \bar{t}$ production differential cross-sections are measured as a function of observables involving the top quark and the $t \bar{t}$ system, a dedicated event reconstruction is performed in each topology. The underlying differential cross-section distributions are obtained from the detector-level events using an unfolding technique that corrects for detector effects. The iterative Bayesian method [3] as implemented in RooUnfold [4] is used.

The differential cross-sections for $t \bar{t}$ production are measured as a function of a large number of variables ( $>60$ spectra in total) including, for the first time in this channel in ATLAS, double-differential distributions. Moreover, the amount of data and the reduced detector uncertainties compared to previous publications also allows double differential measurements in the boosted topology to be made for the first time.

The analysis investigates a list of variables that characterise various aspects of the $t \bar{t}$ system production. In particular, the variables selected are sensitive to the kinematics of the top and anti-top quarks and of the $t \bar{t}$ system or are sensitive to initial- and finalstate radiation effects. Furthermore, the variables are sensitive to the differences among parton density functions (PDFs) and possible effects beyond the SM. Both normalised and absolute differential cross-sections are measured, with more emphasis given to the discussion of the normalised results.

The particle-level measurements are compared with next-to-leading order plus parton shower (NLO+PS) Monte Carlo (MC) predictions as implemented in state-ofthe-art MC generators. At the particle level, the predictions agree with the singledifferential measurements over a wide kinematic region for both the resolved and boosted topologies, although poorer modelling is observed in specific regions of the probed phasespace, as shown in Figure 1.

In the boosted topology, which is focused in the region where the hadronic top quark is produced with high $p_{\mathrm{T}}$, a disagreement between the measured inclusive crosssection and several predictions is observed, as shown in Figure 2. Overall, the NLO+PS $\mathrm{MC}$ generators show $t \bar{t}$ poorer modelling of the double-differential distributions and no combination that includes $p_{\mathrm{T}}$ can be described by the generators in the resolved topology.

Overall, the POWHEG [20]+PYTHIA8 [21] generator and, in the boosted topology, POWHEG+HERWIG7 [22] are the two generators able to give a good prediction of the 
largest fraction of the probed variables. The measurements show high sensitivity to the different aspects of the predictions of the MC generators. They are hence relevant for the tuning of the MC generators and will contribute to an improved description of the $t \bar{t}$ final state and to reducing the systematic uncertainties related to top-quark modelling.

The measured parton-level differential cross-sections are compared with state-ofthe-art fixed-order next-to-next-to-leading order (NNLO) QCD predictions [23]. A general improvement relative to the $\mathrm{NLO}+\mathrm{PS} \mathrm{MC}$ generators is found in the level of agreement of the single- and double-differential cross-sections in both the resolved and boosted regimes. The comparison of double-differential distributions with NNLO predictions provides a very stringent test of the SM description of $t \bar{t}$ production. The comparison with the NNLO pQCD predictions including EW corrections [24], due to the still rather limited range probed for the measured transverse momenta of the top and anti-top quarks, does not yet allow the impact of the EW corrections in the production of top-quark pairs to be quantified. The measured differential cross-sections at the parton level will be able to be used in detailed phenomenological studies and in particular to improve the determination of the gluon density in the proton and of the top-quark pole mass.

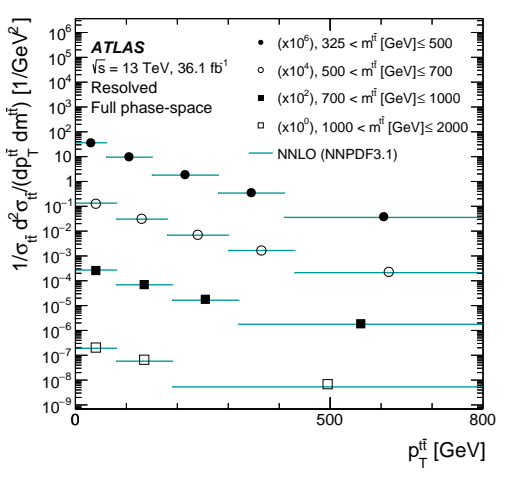

(a)

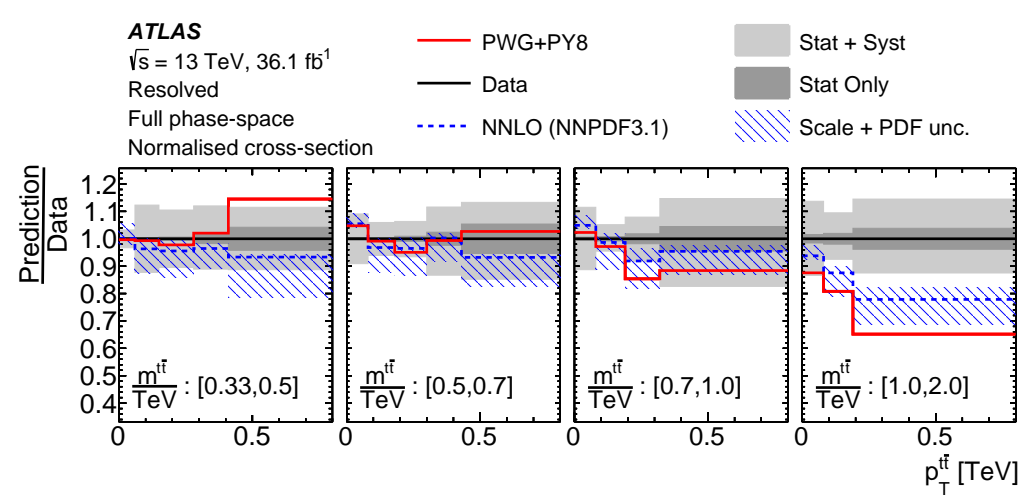

(b)

Figure 1: (a) Parton-level normalised differential cross-section as a function of $p_{\mathrm{T}}^{t \bar{t}}$ in bins of $m^{t \bar{t}}$ in the resolved topology compared with the NNLO prediction obtained using the NNPDF3.1 NNLO PDF set [25]. Data points are placed at the centre of each bin. (b) The ratio of the measured cross-section to the NNLO prediction and the prediction obtained with the POWHEG+PYTHIA8 MC generator. The hatched band represents the total uncertainty in the NNLO prediction. The solid bands represent the statistical and total uncertainty in the data. [2]

\subsection{Measurement of the $t \bar{t}$ production cross-section and lepton differential

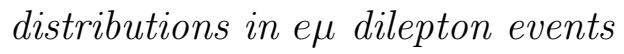

The opposite-sign $e \mu$ plus $b$-tagged jets signature allows precise measurements of the differential distributions of the leptons produced in $t \bar{t}$ events to be made. 


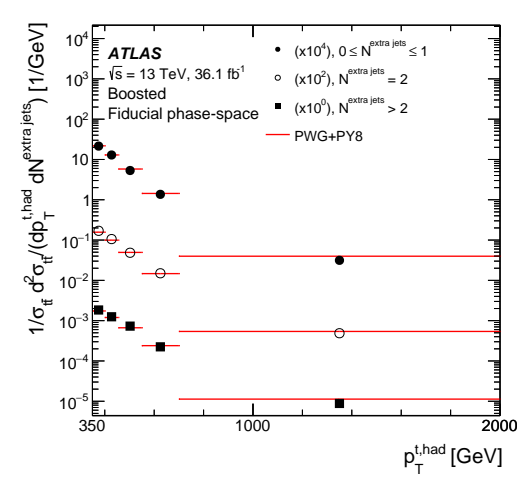

(a)

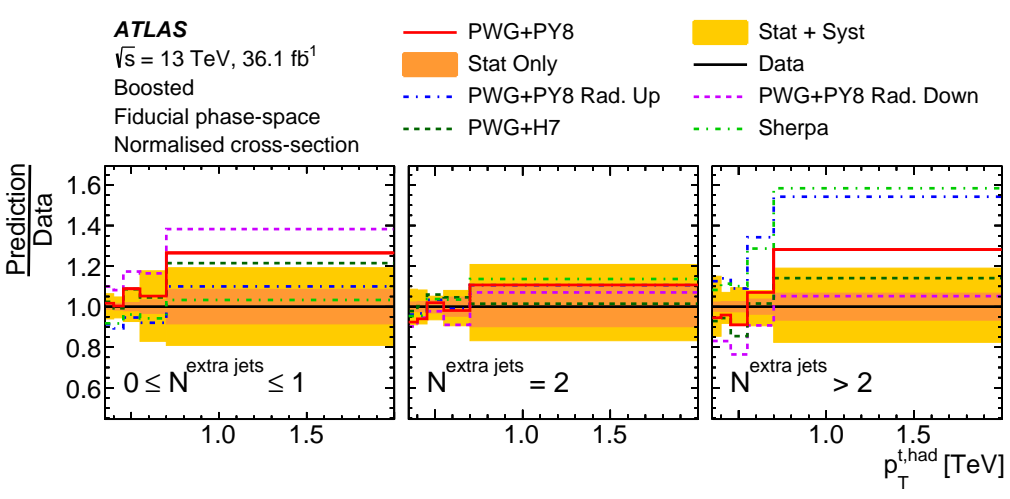

(b)

Figure 2: (a) Particle-level normalised differential cross-section as a function of the $p_{\mathrm{T}}$ of the hadronically decaying top quark in bins of the number of additional jets in the boosted topology compared with the prediction obtained with the POWHEG+PYTHIA8 MC generator. Data points are placed at the centre of each bin. (b) The ratio of the measured cross-section to different MC predictions. The bands represent the statistical and total uncertainty in the data. [2]

The cross-section is measured, at $13 \mathrm{TeV}$ with an integrated luminosity of $36.1 \mathrm{fb}^{-1}$, to be $\sigma_{t \bar{t}}=826.4 \pm 3.6$ (stat) \pm 11.5 (syst) \pm 15.7 (lumi) \pm 1.9 (beam) pb [5], where the four uncertainties are due to data statistics, experimental and theoretical systematic effects, integrated luminosity, and LHC beam energy, giving a total uncertainty of $2.4 \%$. The result is consistent with state-of-the-art theoretical QCD calculations and represents the most precise cross section measurement at the moment. The measurement is used to determine the top quark pole mass via the dependence of the predicted crosssection on $m_{t}^{\text {pole }}$ resulting in $m_{t}^{\text {pole }}=173.1_{-2.1}^{+2.0} \mathrm{GeV}$. It is also combined with previous measurements at $\sqrt{s}=7 \mathrm{TeV}$ and $\sqrt{s}=8 \mathrm{TeV}$, as shown in Figure 3, to derive ratios and double ratios of $t \bar{t}$ and $Z$ cross-sections at different energies.

This paper also presents the measurements of the absolute and normalised differential cross-sections as functions of the transverse momentum $p_{\mathrm{T}}^{\ell}$ and absolute pseudorapidity $\left|\eta^{\ell}\right|$ of the single leptons, the $p_{\mathrm{T}}$, invariant mass and absolute rapidity of the $e \mu$ system, the absolute azimuthal angle $|\Delta \phi|$ between the two leptons in the transverse plane, and the scalar sums of the momenta and energies of the two leptons. These distributions are found to be generally well described by predictions from a variety of leading order (LO) multileg and next-to-leading order (NLO) $t \bar{t}$ matrix-element event generators interfaced to parton showers, and by NLO fixed-order QCD calculations. The sensitivity of the data to the gluon PDF and to the top quark pole mass is also shown. Two-dimensional distributions of $\left|\eta^{\ell}\right|,\left|y^{e \mu}\right|$ and $\left|\Delta \phi^{e \mu}\right|$ as functions of $m^{e \mu}$ are also reported. The data are again compared to the predictions of various NLO $t \bar{t}$ matrixelement event generators, but the interpretations in terms of PDF constraints and $m^{\text {pole }}$ are left for future work. 


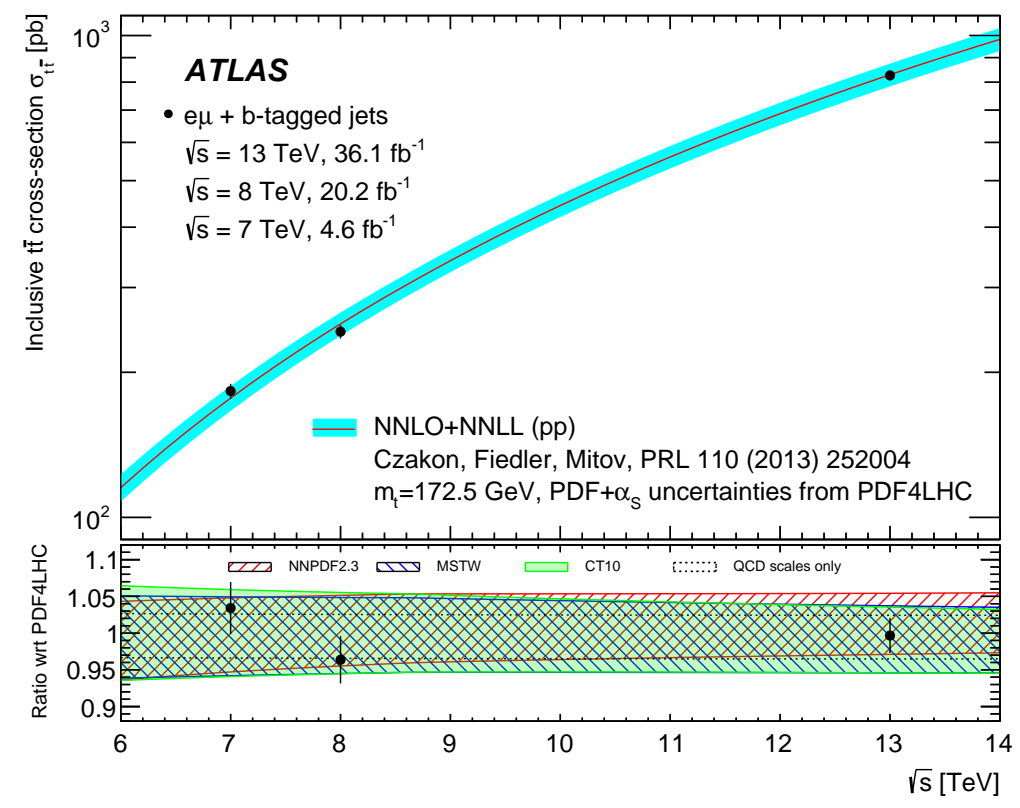

Figure 3: Inclusive $t \bar{t}$ cross-section $\sigma_{t \bar{t}}$ as a function of centre-of-mass energy, comparing ATLAS results from the $e \mu$ plus $b$-tagged jets final state at $\sqrt{s}=7,8$ and $13 \mathrm{TeV}$ with NNLO+NNLL theoretical predictions using the PDF4LHC prescription for PDF and $\alpha_{s}$ uncertainties, and $m_{t}=172.5 \mathrm{GeV}$. The lower plot shows the ratios of the measurements and predictions to the central value of the prediction using PDF4LHC. The total uncertainties when using the individual NNPDF2.3, MSTW and CT10 PDFs are shown as overlapping hatched or coloured bands, and the dotted lines show the QCD scale uncertainties alone. The $\sqrt{s}=7 \mathrm{TeV}$ and $\sqrt{s}=8 \mathrm{TeV}$ results are taken from Ref. [6], with the LHC beam energy uncertainties reduced according to Ref. [7]. [5]

\section{Top-quark mass measurements}

\subsection{Measurement of the top-quark mass in $t \bar{t}+1$-jet events at $\sqrt{s}=8 \mathrm{TeV}$}

The measurement takes advantage of the sensitivity to the top-quark mass of the differential cross section of $t \bar{t}$ production in association with at least one energetic jet. The presence of the additional jet enhances the sensitivity to the top-quark mass in comparison with similar observables defined for the $t \bar{t}$ system only. In particular, the observable used to extract the top-quark mass, $R$, is defined as the normalised differential $t \bar{t}+1$ jet cross section, as a function of the parameter $\rho_{S}=\frac{2 m_{0}}{m_{t \bar{t}+1 \text { jet }}}$, with $m_{0}$ representing a constant fixed to $170 \mathrm{GeV}$ and $m_{t \bar{t}+1}$ jet representing the invariant mass of the $t \bar{t}+1$ jet system.

The measurement [8] is performed using $20.2 \mathrm{fb}^{-1}$ collision data produced by the LHC and collected by the ATLAS experiment. The normalised differential cross section of top-quark pair production in association with an energetic jet is measured in the lepton+jets final state and unfolded to parton and particle levels. The unfolded 
distribution at parton level can be described using NLO QCD predictions in terms of either the top-quark pole mass or the running mass as defined in the (modified) minimal subtraction scheme $(\overline{\mathrm{MS}})$. A comparison between the experimental distribution and the theoretical prediction allows the top-quark mass to be extracted in the two schemes. The value obtained for the pole-mass scheme is $m_{t}^{\text {pole }}=171.1 \pm 0.4($ stat $) \pm$ 0.9 (syst) ${ }_{-0.3}^{+0.7}$ (theo) GeV. The extracted value in the running-mass scheme is $m_{t}\left(m_{t}\right)=$ $162.9 \pm 0.5$ (stat) \pm 1.0 (syst) ${ }_{-1.2}^{+2.1}$ (theo) $\mathrm{GeV}$. The results for the top-quark mass using the two schemes are consistent, when translated from one scheme to the other.

Figure 4 shows the summary of top-quark pole mass measurements: it represents the best individual differential measurement, with a relative uncertainty of $0.7 \%$, mainly due to jet-energy-scale and MC modelling uncertainties.

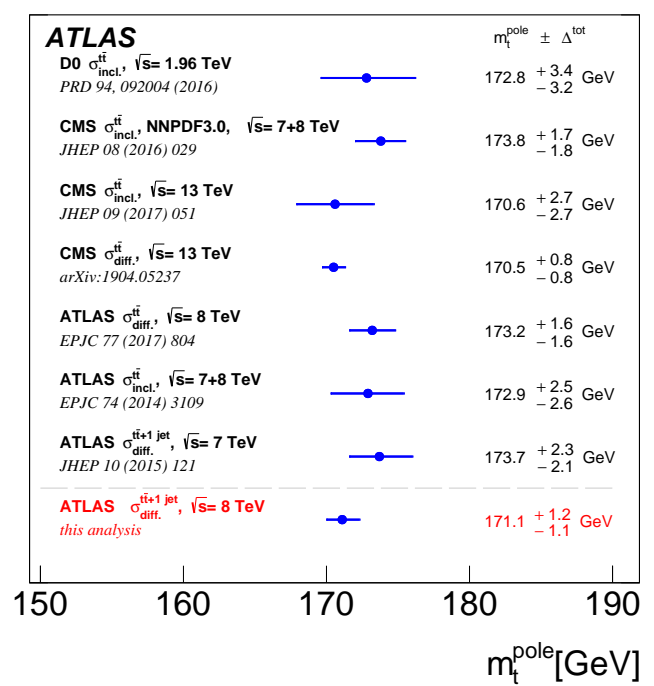

Figure 4: Summary of top-quark pole mass measurements at the Tevatron and the LHC. [8]

\subsection{Measurement of the top quark mass in the $t \bar{t} \rightarrow$ lepton + jets channel at} $\sqrt{s}=8 \mathrm{TeV}$ and combination with previous results

The top quark mass is measured using a template method in the $t \bar{t} \rightarrow$ lepton + jets channel (lepton is $e$ or $\mu$ ) using ATLAS data recorded in 2012 at the LHC, at a proton-proton centre-of-mass energy of $\sqrt{s}=8 \mathrm{TeV}$ and corresponding to an integrated luminosity of $20.2 \mathrm{fb}^{-1}[9]$.

The $t \bar{t} \rightarrow$ lepton + jets channel is characterized by the presence of a charged lepton, a neutrino and four jets, two of which originate from bottom quarks $(b)$. Exploiting a three-dimensional template technique, the top quark mass is determined together with a global jet energy scale factor and a relative b-to-light-jet energy scale factor. The mass of the top quark is measured to be $m_{t}=172.08 \pm 0.39$ (stat) \pm 0.82 (syst) GeV. 
A combination with previous ATLAS measurements gives $m_{t}=172.69 \pm 0.25$ (stat) \pm 0.41 (syst) $\mathrm{GeV}$, which corresponds to a relative uncertainty of $0.29 \%$.

Figure 5 compares the top masses from different experiments.

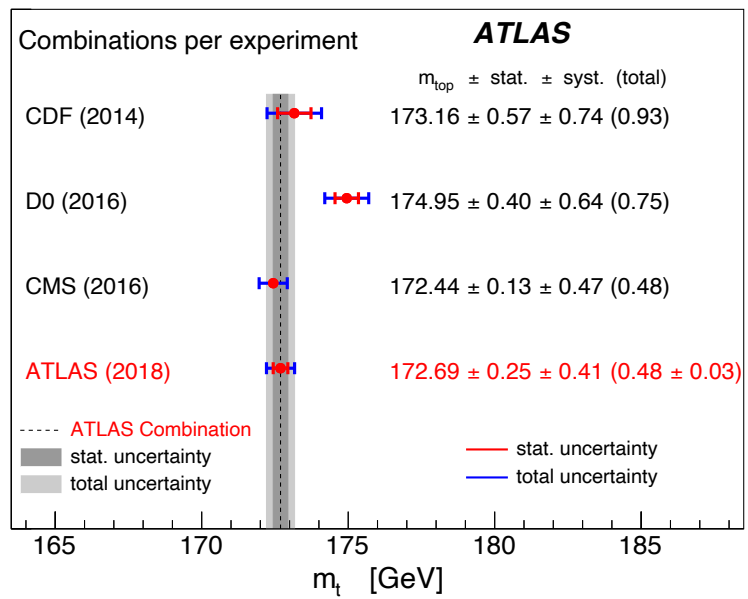

Figure 5: The most precise result of $m_{t}$ per experiment from the latest combinations performed by the individual experiments. The vertical band corresponds to the new ATLAS combined value of $m_{t}$. The values quoted are the combined value, the statistical uncertainty, the systematic uncertainty, the total uncertainty and for ATLAS results also the uncertainty in the total uncertainty, which is statistical. [9]

\section{Top properties measurements}

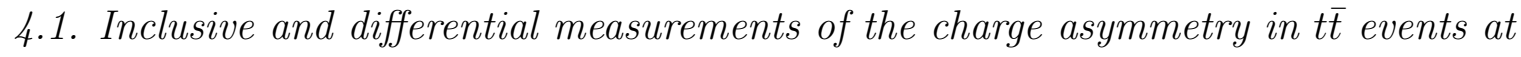
$13 \mathrm{TeV}$

Production of top quark pairs is symmetric at leading-order (LO) under charge conjugation. The asymmetry between the $t$ and $\bar{t}$ originates from interference of the higher-order amplitudes in the $q \bar{q}$ and $q g$ initial states, with the $q \bar{q}$ annihilation contribution dominating. The contribution from electro-weak corrections is about $13 \%$ for the inclusive asymmetry. The $q g \rightarrow t \bar{t} g$ production process is also asymmetric, but its cross section is much smaller than $q \bar{q}$. Gluon fusion production is symmetric to all orders. As a consequence of these asymmetries, the top quark is preferentially produced in the direction of the incoming quark.

At a $p \bar{p}$ collider, where the preferential direction of the incoming quark (antiquark) always almost coincides with that of the proton (anti-proton), a forward-backward asymmetry $A_{\mathrm{FB}}$ can be measured directly. At the LHC $p p$ collider, since the colliding beams are symmetric, it is not possible to measure $A_{\mathrm{FB}}$ as there is no preferential direction of either the top quark or the top antiquark. However, due to the difference in the proton parton distribution functions, on average the valence quarks carry a larger 
fraction of the proton momentum than the sea antiquarks. This results in more forward top quarks and more central top antiquarks.

A central-forward charge asymmetry for the $t \bar{t}$ production, referred to as the charge asymmetry $\left(A_{\mathrm{C}}\right)$, is defined as $A_{\mathrm{C}}^{t \bar{t}}=\frac{N(\Delta|y|>0)-N(\Delta|y|<0)}{N(\Delta|y|>0)+N(\Delta|y|<0)}$, where $\Delta|y|=|y(t)|-|y(\bar{t})|$ is the difference between the absolute value of the top-quark rapidity $\left|y_{t}\right|$ and the absolute value of the top-antiquark rapidity $\left|y_{\bar{t}}\right|$.

The measurement of the $t \bar{t}$ charge asymmetry is performed using data corresponding to an integrated luminosity of $139 \mathrm{fb}^{-1}$ from the ATLAS experiment [10]. It is performed in the single-lepton channel combining both the resolved and boosted topologies of top quark decays. A Bayesian unfolding procedure is used to infer the asymmetry at parton level, correcting for detector resolution and acceptance effects.

The inclusive $t \bar{t}$ charge asymmetry is measured as $A_{\mathrm{C}}=0.0060 \pm 0.0015$ (stat+syst.), which differs from zero by 4 standard deviations. It corresponds to the first evidence for charge asymmetry in $p p$ collisions. Differential measurements are performed as a function of the invariant mass and longitudinal boost of the $t \bar{t}$ system. Both inclusive and differential measurements are found to be compatible with the SM predictions, at NNLO in perturbation theory with NLO electroweak corrections, and are shown in Figure 6.

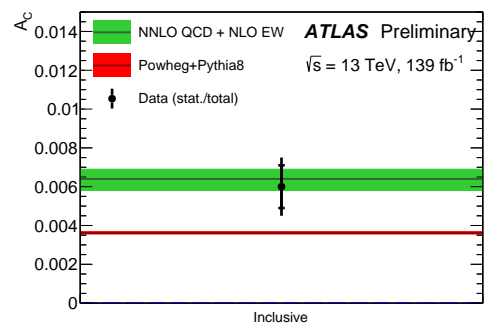

(a)

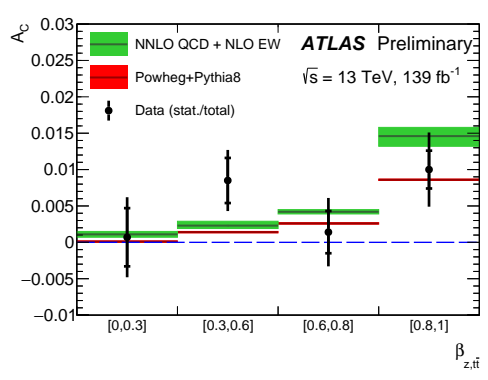

(b)

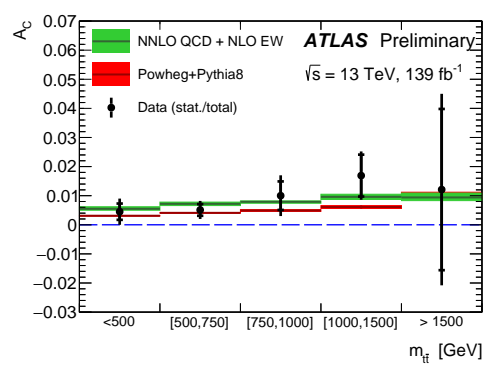

(c)

Figure 6: The unfolded inclusive (a) and differential charge asymmetries as a function of the invariant mass (b) and the longitudinal boost (c) of the top pair system in data (resolved and boosted topologies are combined). Green hatched regions show SM theory predictions calculated at NNLO in QCD and NLO in electroweak theory. Red hatched regions show parton-level truth asymmetry with its uncertainty extracted from the full phase space using the nominal $t \bar{t}$ signal sample. Vertical bars correspond to the total uncertainties. [10]

4.2. Measurement of the top-quark decay width in top-quark pair events in the dilepton channel at $\sqrt{s}=13 \mathrm{TeV}$

In the SM, at leading order (LO) in perturbation theory, the top-quark decay width, $\Gamma_{t}$, depends on the top-quark mass $m_{t}$, the Fermi coupling constant $G_{\mathrm{F}}$ and the CKM matrix element $\left|V_{t b}\right|$. The most precise theoretical predictions at NNLO yield $\Gamma_{t}=$ 
$1.322 \mathrm{GeV}$ for a top-quark mass of $172.5 \mathrm{GeV}$ [12]. The uncertainty on the theoretical prediction of the top-quark decay width from variations of the renormalisation scale is $<1 \%$. Propagating the experimental uncertainties to the parameters that enter the calculation increases the uncertainty up to $\sim 6 \%$. Possible deviations from the expected value of the top-quark decay width could hint at non-SM decay channels of the top quark or modification of top-quark couplings (e.g., existence of a charged Higgs bosons or Flavour Changing Neutral Current (FCNC) processes).

$\Gamma_{t}$ can be measured either directly or indirectly. Indirect measurements rely on some specific predictions of the SM, e.g. the theoretical SM calculation of the single-top-quark cross-section and theoretical calculations of the partial top-quark decay width. This analysis [11], instead uses a direct approach to measure $\Gamma_{t}$. It is based on data collected at a centre-of-mass energy of $\sqrt{s}=13 \mathrm{TeV}$, recorded with the ATLAS detector, and focuses on $t \bar{t}$ events in the dilepton decay channel. This topology results in a signature of two $b$-jets, two charged leptons and large missing transverse momentum, due to the two neutrinos. The invariant mass of a charged lepton and a $b$-jet, $m_{\ell b}$, from $e \mu$ events is reconstructed and used in a profile-likelihood fit with simulated templates for various top-quark decay widths to extract the width in data. Systematic uncertainties are incorporated into the fit and are constrained by simultaneously fitting the invariant mass of the two $b$-jets, $m_{b \bar{b}}$, from combined $e e$ and $\mu \mu$ events. The measured top-quark decay width is $\Gamma_{t}=1.9 \pm 0.5 \mathrm{GeV}$ for a top-quark mass of $m_{t}=172.5 \mathrm{GeV}$, in agreement with the SM prediction.

\subsection{Measurements of top-quark pair spin correlations in the e $\mu$ channel at $\sqrt{s}=13 \mathrm{TeV}$}

Due to the unstable nature of top quarks, their spin information is accessed through their decay products. However, not all decay particles carry the spin information to the same degree, with charged leptons arising from leptonically decaying $W$ bosons carrying almost the full spin information of the parent top quark. This feature, along with the fact that charged leptons are readily identified and reconstructed by collider experiments, means that observables to study spin correlation in $t \bar{t}$ events are often based on the angular distributions of the charged leptons in events where both $W$ bosons decay leptonically (referred to as the dilepton channel). The simplest observable is the absolute azimuthal opening angle between the two charged leptons, measured in the laboratory frame in the plane transverse to the beam line. This opening angle is denoted by $\Delta \phi$.

The measurement of observables sensitive to spin correlations in $t \bar{t}$ production uses $36.1 \mathrm{fb}^{-1}$ of $p p$ collision data at $\sqrt{s}=13 \mathrm{TeV}$ recorded with the ATLAS detector [13]. Differential cross-sections are measured in events with exactly one electron and one muon with opposite-sign electric charge as a function of the azimuthal opening angle $\Delta \phi$ and the absolute difference in pseudorapidity $|\Delta \eta|$ between the electron and muon candidates in the laboratory frame. The azimuthal opening angle is also measured as a function of 
the invariant mass of the $t \bar{t}$ system. The measured differential cross-sections, unfolded to fiducial particle level and full phase-space parton level, are compared to predictions by several NLO MC generators and fixed-order calculations.

None of the studied generators are able to reproduce the normalised $\Delta \phi$ distribution within the experimental errors. A comparison was made with fixed-order predictions at NNLO in QCD and in an expansion (of the normalised differential distribution in powers of the couplings) at NLO in QCD and EW couplings with a fixed scale choice. The former slightly improves the description of the data, while the latter describes the data but with large scale uncertainties (Figure 7).

The discrepancy remains unresolved, but is stimulating state-of-the-art predictions and development in the theory community [14].

Using these leptonic observables, a search is performed for pair production of supersymmetric top squarks decaying into SM top quarks and light neutralinos. Top squark masses between 170 and $230 \mathrm{GeV}$ are largely excluded at the $95 \%$ confidence level for kinematically allowed values of the neutralino mass.

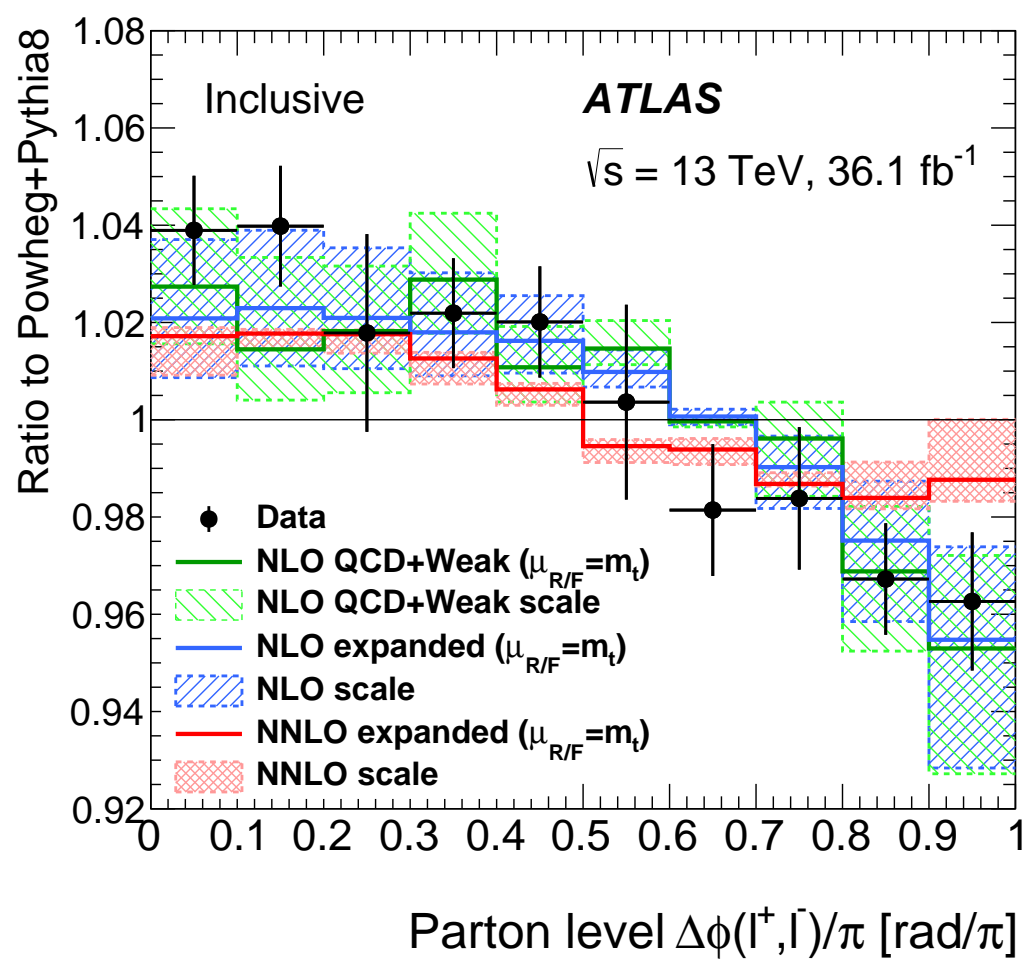

Figure 7: Comparison of the unfolded $\Delta \phi$ distribution with theoretical predictions for the inclusive selection: ratio as compared with POWHEG + PYTHIA8 for NLO and NNLO theoretical predictions. [13] 
4.4. Combinations of single-top-quark production cross-section measurements and $\left|f_{\mathrm{LV}} V_{t b}\right|$ determinations at $\sqrt{s}=7$ and 8 TeV with the ATLAS and CMS experiments

The cross-section measurements by the ATLAS and CMS Collaborations, using data from LHC $p p$ collisions corresponding to integrated luminosities of 1.17 to $5.1 \mathrm{fb}^{-1}$ at $\sqrt{s}=7 \mathrm{TeV}$, and 12.2 to $20.3 \mathrm{fb}^{-1}$ at $\sqrt{s}=8 \mathrm{TeV}$, are combined for the single-topquark production modes ( $t$-, $t W$-, and $s$-channel) [15]. The combined $t$-channel crosssections are $67.5 \pm 5.7 \mathrm{pb}$ and $87.7 \pm 5.8 \mathrm{pb}$ at $\sqrt{s}=7$ and $8 \mathrm{TeV}$, respectively. The combined $t W$-channel cross-sections are $16.3 \pm 4.1 \mathrm{pb}$ and $23.1 \pm 3.6 \mathrm{pb}$ at $\sqrt{s}=7$ and $8 \mathrm{TeV}$, respectively. For the $s$-channel cross-section, the combination yields $4.9 \pm 1.4 \mathrm{pb}$ at $\sqrt{s}=8 \mathrm{TeV}$.

The square of the magnitude of the CKM matrix element $V_{t b}$ multiplied by a form factor $f_{\mathrm{LV}}$ is determined for each production mode and centre-of-mass energy, using the ratio of the measured cross-section to its theoretical prediction. It is assumed that the top-quark-related CKM matrix elements obey the relation $\left|V_{t d}\right|,\left|V_{t s}\right| \ll\left|V_{t b}\right|$. All the $\left|f_{\mathrm{LV}} V_{t b}\right|^{2}$ determinations, extracted from individual ratios at $\sqrt{s}=7$ and $8 \mathrm{TeV}$, are combined, resulting in $\left|f_{\mathrm{LV}} V_{t b}\right|=1.02 \pm 0.04$ (meas.) \pm 0.02 (theo.). All combined measurements are consistent with their corresponding SM predictions.

Two summary plots are shown in Figures 8.

\section{Measurements related to the associated production of a $t \bar{t}$ quark pair with additional heavy-flavour jets at $\sqrt{s}=13 \mathrm{TeV}$}

Measurements of the production cross-section of $t \bar{t}$ pairs with additional jets provide important tests of QCD predictions. Among these, the process of $t \bar{t}$ produced in association with jets originating from $b$-quarks ( $b$-jets) is particularly important to measure, as there are many uncertainties in the calculation of the process.

A measurement of such a production process in $p p$ collisions at the LHC at a centreof-mass energy of $\sqrt{s}=13 \mathrm{TeV}$ is performed, using data recorded with the ATLAS detector and corresponding to an integrated luminosity of $36.1 \mathrm{fb}^{-1}$ [16]. Fiducial crosssection measurements are performed in the dilepton and lepton-plus-jets decay channels and presented at particle level in the form of inclusive cross-sections of $t \bar{t}$ final states with three and four $b$-jets as well as differential cross-sections as a function of global event properties and properties of $b$-jet pairs.

As shown in Figure 9, the measured inclusive fiducial cross-sections generally exceed the $t \bar{t} b \bar{b}$ predictions from various NLO matrix element calculations matched to a parton shower but are compatible within the total uncertainties. The experimental uncertainties are smaller than the uncertainties in the predictions. Comparisons of state-of-the-art theoretical predictions with the differential measurements are shown and found in good agreement with data for most of them. 


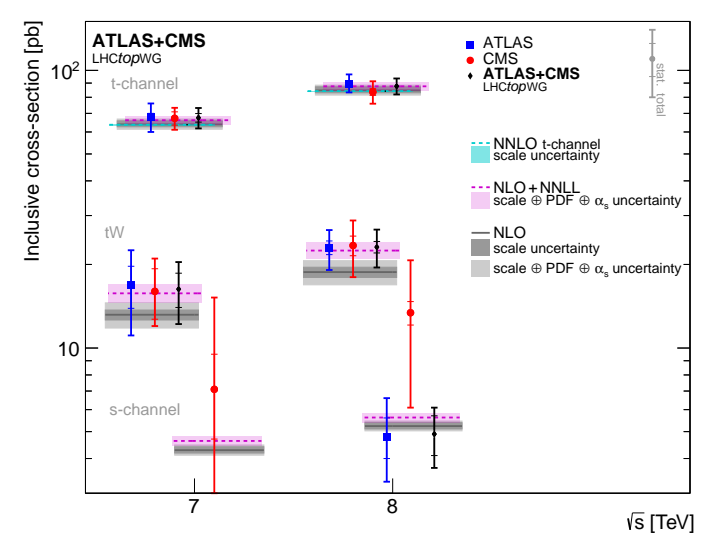

(a)

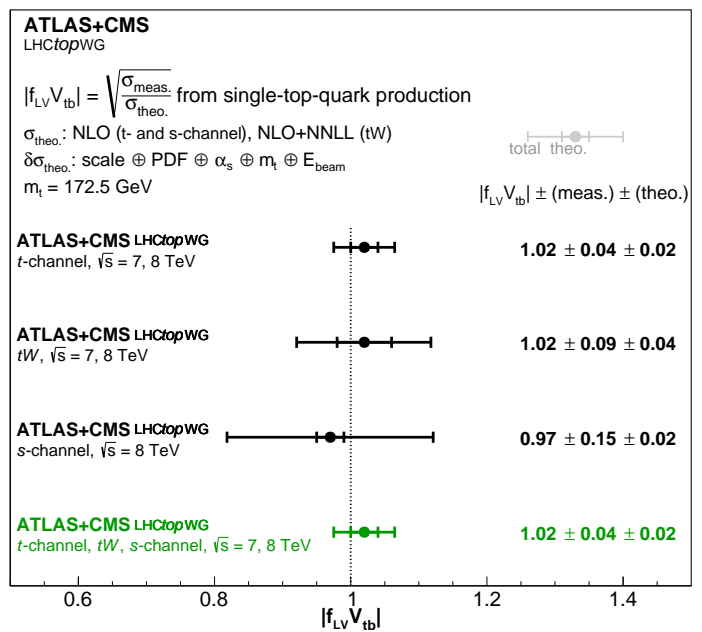

(b)

Figure 8: (a) Single-top-quark cross-section measurements performed by ATLAS and CMS, together with the combined results. These measurements are compared with the theoretical predictions at NLO and NLO+NNLL for all three production modes and the prediction at NNLO for $t$-channel only. The corresponding theoretical uncertainties are also presented. The scale uncertainty for the NNLO prediction is small and is presented as a narrow band under the dashed line. (b) The combined $\left|f_{\mathrm{LV}} V_{t b}\right|$ value extracted from the $t$-channel and $t W$ cross-section measurements at $\sqrt{s}=7$ and $8 \mathrm{TeV}$ from ATLAS and CMS, as well as the ATLAS s-channel measurement at $\sqrt{s}=8 \mathrm{TeV}$, is shown together with the combined $\left|f_{\mathrm{LV}} V_{t b}\right|$ values for each production mode. The theoretical predictions for $t$-channel and $s$-channel production are computed at NLO accuracy, while the theoretical predictions for $t W$ are calculated at NLO+NNLL accuracy. The $\sigma_{\text {theo. }}$ uncertainties used to compute $\left|f_{\mathrm{LV}} V_{t b}\right|$ include scale, $\mathrm{PDF}+\alpha_{s}, m_{t}$, and $E_{\text {beam }}$ variations. [15]

\section{Search of new physics involving top quark production}

\subsection{Search for top-quark decays $t \rightarrow H q$ at $\sqrt{s}=13 \mathrm{TeV}$}

In the SM, FCNC decays are suppressed relative to the dominant $t \rightarrow W b$ decay mode, since $t q H$ interactions are forbidden at the tree level and suppressed even at higher orders in the perturbative expansion due to the Glashow-Iliopoulos-Maiani (GIM) mechanism. As a result, the SM predictions for the $t \rightarrow H q$ branching ratios are exceedingly small, $B R(t \rightarrow H u) \sim 10^{-17}$ and $B R(t \rightarrow H c) \sim 10^{-15}$, making them undetectable in the foreseeable future. In contrast, large enhancements of these branching ratios are possible in some scenarios beyond the SM (e.g., quark-singlet models, two-Higgs-doublet models (2HDM), supersymmetric models with $R$-parity violation, composite Higgs models). In these scenarios, branching ratios can be as high as $B R(t \rightarrow H q) \sim 10^{-3}$ (2HDM Type III scenario).

The search for FCNC decays $t \rightarrow H q$ is based on a dataset of $p p$ collisions at 

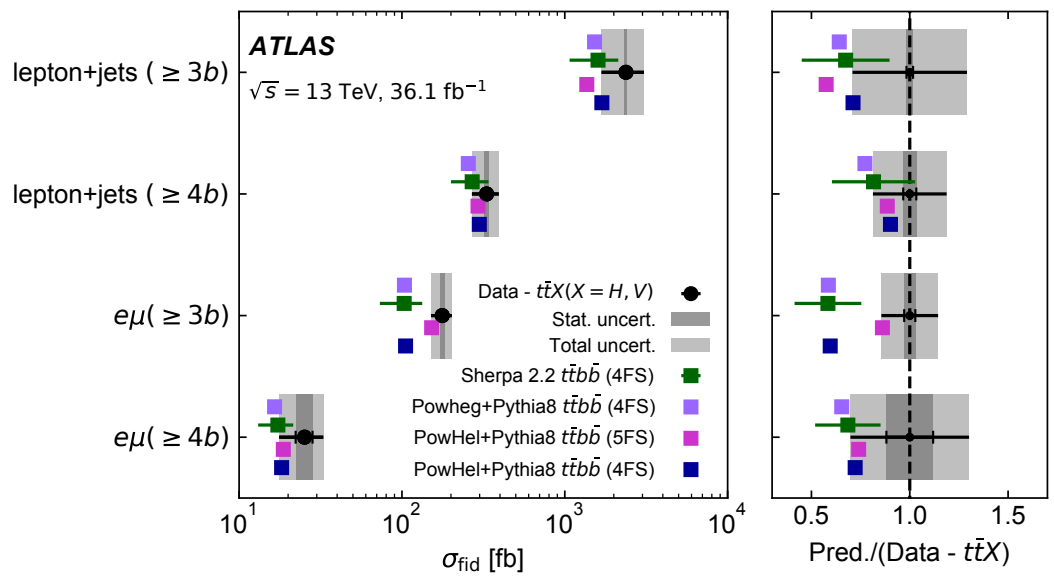

Figure 9: The measured fiducial cross-sections, with $t \bar{t} H$ and $t \bar{t} V$ contributions subtracted from data, compared with $t \bar{t} b \bar{b}$ predictions obtained using the SHERPA 2.2 generator with uncertainties obtained by varying the renormalisation and factorisation scales by factors of 0.5 and 2.0 and including PDF uncertainties. Comparisons with the central values of the predictions of POWHEG+PYTHIA 8 and PowHel+PYTHIA $8 t \bar{t} b \bar{b}$ are also made. No uncertainties are included in the subtraction of the $t \bar{t} H$ or $t \bar{t} V$ predictions. [16]

$\sqrt{s}=13 \mathrm{TeV}$ recorded with the ATLAS detector and corresponding to an integrated luminosity of $36.1 \mathrm{fb}^{-1}$ [17]. Two complementary analyses are performed to search for top-quark pair events in which one top quark decays into $W b$ and the other top quark decays into $H q$, and target the $H \rightarrow b \bar{b}$ and $H \rightarrow \tau \tau$ decay modes, respectively. The high multiplicity of $b$-quark jets, or the presence of hadronically decaying $\tau$-leptons, is exploited in the two analyses respectively. Multivariate techniques are used to separate the signal from the background, which is dominated by top-quark pair production.

No significant excess of events above the background expectation is found, and 95\% CL upper limits on the $t \rightarrow H q$ branching ratios are derived. The combination of these searches with ATLAS searches in diphoton and multilepton final states yields observed (expected) 95\% CL upper limits on the $t \rightarrow H c$ and $t \rightarrow H u$ branching ratios of $1.1 \times 10^{-3}\left(8.3 \times 10^{-4}\right)$ and $1.2 \times 10^{-3}\left(8.3 \times 10^{-4}\right)$, respectively. The corresponding combined observed (expected) upper limits on the $\left|\lambda_{t c H}\right|$ and $\left|\lambda_{t u H}\right|$ couplings are 0.064 (0.055) and $0.066(0.055)$, respectively.

6.2. Search for flavour-changing neutral currents in processes with one top quark and a photon using $81 \mathrm{fb}^{-1}$ at $\sqrt{s}=13 \mathrm{TeV}$

This paper presents a search for FCNCs in processes with a top quark and a photon based on data collected with the ATLAS experiment at $\sqrt{s}=13 \mathrm{TeV}$ [18]. The analysis is most sensitive to the production of a single top quark plus a photon, but also considers the decay of a pair-produced top quark into an up or charm quark plus a photon. Events 
with a photon, an electron or muon, a $b$-tagged jet, and missing transverse momentum are selected. A neural network based on kinematic variables differentiates between events from signal and background processes. The data are consistent with the backgroundonly hypothesis, and limits are set on the strength of the $t q \gamma$ coupling in an effective field theory. These are also interpreted as 95\% CL upper limits on the cross section for FCNC $t \gamma$ production via a left-handed (right-handed) tu $\gamma$ coupling of $36 \mathrm{fb}$ (78 fb) and on the branching ratio for $t \rightarrow \gamma u$ of $2.8 \times 10^{-5}\left(6.1 \times 10^{-5}\right)$. In addition, they are interpreted as $95 \%$ CL upper limits on the cross section for FCNC $t \gamma$ production via a left-handed (right-handed) $t c \gamma$ coupling of $40 \mathrm{fb}(33 \mathrm{fb})$ and on the branching ratio for $t \rightarrow \gamma c$ of $22 \times 10^{-5}\left(18 \times 10^{-5}\right)$.

\section{Measurement of strange hadron production from $t \bar{t}$ pair decays}

The production of $K_{S}^{0}$ and $\Lambda^{0}$ hadrons in $t \bar{t}$ final states has been studied on a data sample with integrated luminosity of $4.6 \mathrm{fb}^{-1}$, at a centre-of-mass energy of $7 \mathrm{TeV}$ with the ATLAS detector [19]. Neutral strange particles are separated into three classes, depending on whether they are contained in a jet, with or without a $b$-tag, or not associated with a selected jet. The aim is to look for differences in their main kinematic distributions. A comparison of data with several MC simulations using different hadronisation and fragmentation schemes, colour reconnection models and different tunes for the underlying event has been made. The production of neutral strange particles in $t \bar{t}$ dileptonic events is found to be well described by current MC models for $K_{S}^{0}$ and $\Lambda^{0}$ production within jets, but not for those produced outside jets, as shown in Figure 10.

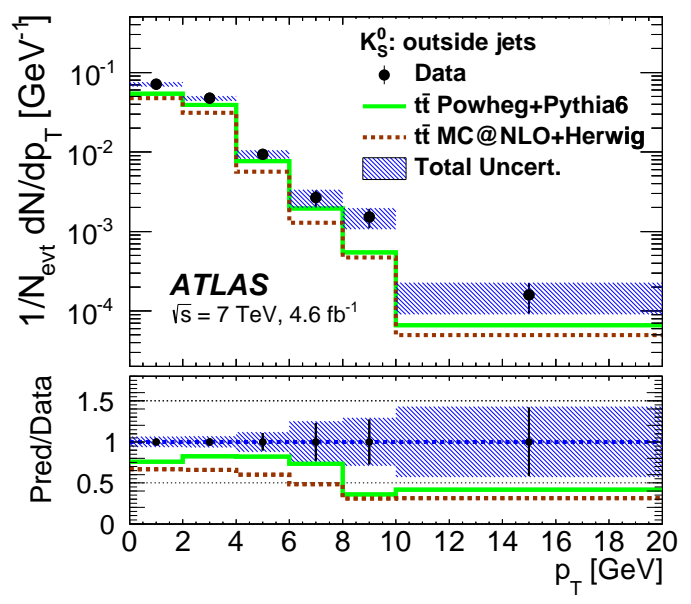

Figure 10: Kinematic characteristics for $K_{S}^{0}$ production not associated with jets, for data and detector-level MC events simulated with the POWHEG+PYTHIA6 and MC@NLO+HERWIG generators. Total uncertainties are represented by the shaded area. Statistical uncertainties for MC samples are negligible in comparison with data. [19] 


\section{Conclusions and outlook}

The latest results in the top-quark sector with the ATLAS experiment at the LHC have been presented.

The one- and two-dimensional differential cross-section measurements for the production of top-antitop pairs confirm the SM NLO predictions with high precision, for a large number of observables. A precision of less than $3 \%$ has been reached in the measurement of the $t \bar{t}$ production cross section, better than the NNLO predictions.

Top-quark properties are measured with ever-increasing precision. Different techniques are employed to measure the top-quark mass: an uncertainty of less than $2 \mathrm{GeV}(0.7 \%)$ has been achieved for direct measurements of the pole mass, making it a very competitive method. The first evidence of non-zero top charge asymmetry at the LHC has been obtained: the inclusive $t \bar{t}$ charge asymmetry is measured as $A_{\mathrm{C}}=0.0060 \pm 0.0015$ (stat+syst.), which differs from zero by 4 standard deviations. The first top width measurement at $13 \mathrm{TeV}$ has been performed, with a result that is already competitive with the pole-mass uncertainty. Some tensions between data and

MC predictions for the $t \bar{t}$ spin-correlation measurement triggered lots of stimulating discussions and development in the theory community. Several NLO and NNLO MC models were investigated, but the discrepancy remains unresolved. Single-top quark production measurements in ATLAS and CMS have been combined and the CKM matrix element $\left|V_{t b}\right|$ has been determined with an uncertainty of $4 \%$, in agreement with the SM prediction.

The associated production of $t \bar{t}$ pairs with additional jets provide important tests of QCD predictions: the measured inclusive fiducial cross-sections for $t \bar{t}$ produced in association with jets originating from $b$-quarks ( $b$-jets) exceed the predictions from various NLO calculations, but are compatible within the total uncertainties. This has also triggered new developments to reduce the uncertainties in the theory predictions, which is the most limiting factor in such measurement nowadays.

Finally, one order of magnitude improvement with respect to the previous analysis has been obtained in the search for FCNC $t \gamma$. 


\section{References}

[1] ATLAS Collaboration, 2008 JINST 3 S08003.

[2] ATLAS Collaboration, Eur. Phys. J. C 79 (2019) no.12, 1028

[3] G. D'Agostini, A multidimensional unfolding method based on Bayes' theorem, Nucl. Instrum. Meth. A 362 (1995) 487.

[4] T. Adye, doi:10.5170/CERN-2011-006.313 arXiv:1105.1160 [physics.data-an].

[5] ATLAS Collaboration, arXiv:1910.08819 [hep-ex].

[6] ATLAS Collaboration, Eur. Phys. J. C 74 (2014) no.10, 3109 Addendum: [Eur. Phys. J. C 76 (2016) no.11, 642]

[7] E. Todesco and J. Wenninger, Large Hadron Collider momentum calibration and accuracy, Phys. Rev. Accel. Beams 20 (2017) 081003.

[8] ATLAS Collaboration, JHEP 1911 (2019) 150

[9] ATLAS Collaboration, Eur. Phys. J. C 79 (2019) no.4, 290

[10] ATLAS Collaboration, ATLAS-CONF-2019-026 [https://cds.cern.ch/record/2682109].

[11] ATLAS Collaboration, ATLAS-CONF-2019-038 [https://cds.cern.ch/record/2684952].

[12] J. Gao, C. S. Li and H. X. Zhu, Phys. Rev. Lett. 110 (2013) no.4, 042001

[13] ATLAS Collaboration, Submitted to: Eur.Phys.J. [arXiv:1903.07570 [hep-ex]].

[14] A. Behring, M. Czakon, A. Mitov, A. S. Papanastasiou and R. Poncelet, Phys. Rev. Lett. 123 (2019) no.8, 082001

[15] ATLAS and CMS Collaborations, JHEP 1905 (2019) 088

[16] ATLAS Collaboration, JHEP 1904 (2019) 046

[17] ATLAS Collaboration, JHEP 1905 (2019) 123

[18] ATLAS Collaboration, Phys. Lett. B 800 (2020) 135082

[19] ATLAS Collaboration, Eur. Phys. J. C 79 (2019) no.12, 1017

[20] S. Alioli, P. Nason, C. Oleari and E. Re, JHEP 06 (2010) 043

[21] T. Sjostrand, S. Mrenna and P. Z. Skands, Comput. Phys. Commun. 178 (2008) 852

[22] J. Bellm et al., Eur. Phys. J. C 76 (2016) 196

[23] S. Catani, S. Devoto, M. Grazzini, S. Kallweit and J. Mazzitelli, JHEP 2019 (2019) 100

[24] M. Czakon et al., JHEP 10 (2017) 186

[25] R. D. Ball et al., Eur. Phys. J. C 77 (2017) 663 